\title{
An unusual case of anaplastic lymphoma kinase-positive large B-cell lymphoma in an elderly patient: A case report and discussion
}

\author{
HANZHEN XIONG $^{1}$, SHAO-YAN LIU ${ }^{1}$, YUE-XIN YANG ${ }^{1}$, XUE-XIAN TAN $^{1}$, QIU-PING LUO ${ }^{1}$, \\ JUAN PENG ${ }^{1}$, ZHONG-TANG XIONG $^{1}$, HUI CHEN ${ }^{1}$, JUAN CHEN $^{1}$, ZHI LI $^{2 *}$ and QING-PING JIANG ${ }^{1 *}$
}

\author{
${ }^{1}$ Department of Pathology, The Third Affiliated Hospital of Guangzhou Medical University, Guangzhou, Guangdong 510150; \\ ${ }^{2}$ Department of Pathology, The First Affiliated Hospital of Sun Yat-sen University, Guangzhou, Guangdong 510080, P.R. China
}

Received December 29, 2014; Accepted September 1, 2015

DOI: 10.3892/etm.2016.3129

\begin{abstract}
We present an unusual case of anaplastic lymphoma kinase (ALK)-positive large B-cell lymphoma, with rapid clinical progression, which occurred in a 90 -year-old male patient. The patient presented with numerous enlarged lymph nodes in the neck and mediastinum. Histopathological analysis of a single lymph node detected diffuse large immunoblastic- or plasmablastic-like tumor cells, which were strongly immunoreactive for ALK in a granular cytoplasmic distribution, but negative for the expression of CD20 and CD79a. In addition, polymerase chain reaction assays were unable to detect clonal rearrangements of the $\mathrm{T}$ cell receptor- $\gamma$ and immunoglobulin heavy chain genes in the tumor lesion, and in situ hybridization tested negative for infection with Epstein-Barr virus. The patient underwent a single cycle of chemotherapy using the cyclophosphamide, doxorubicin, vincristine, prednisone, and etoposide (E-CHOP) regimen; however, the patient developed pleural effusions with respiratory distress, associated with clinical deterioration. The patient succumbed to the disease within 4 months of initial presentation. To the best of our knowledge, this is the eldest patient with this type of lymphoma to be reported in the literature.
\end{abstract}

\section{Introduction}

Anaplastic lymphoma kinase-positive large B-cell lymphoma (ALK-positive LBCL) is a rare but distinct lymphoma with a consistent absence of CD20 expression and acquisition of

Correspondence to: Dr Qing-Ping Jiang, Department of Pathology, The Third Affiliated Hospital of Guangzhou Medical University, 63 Duobao Road, Guangzhou, Guangdong 510150, P.R. China E-mail: jiangqp2003@126.com

*Contributed equally

Key words: anaplastic lymphoma kinase, diffuse large B-cell lymphoma, differential diagnosis, prognosis plasma cell markers, including CD38 and CD138. The World Health Organization (WHO) classification of tumors of hematopoietic and lymphoid tissues defined ALK-LBCL as a separate entity to non-Hodgkin's lymphoma, which consists of ALK-positive monomorphic large immunoblast-like B-cells, sometimes with plasmablastic differentiation (1). ALK-positive LBCL appears to be a distinct disease entity, with an aggressive development cycle that is associated with a poor prognosis; the overall median survival of patients with advanced stage III/IV ALK-positive LBCL was 11 months. Previous studies have suggested that this distinct subtype of diffuse large B-cell lymphoma (DLBCL) should belong to the group of pediatric lymphomas $(2,3)$; however, we report an example of ALK-positive LBCL in a 90-year-old male patient, with rapid clinical course. To the best of our knowledge, this is the eldest patient with this type of lymphoma to be reported in the literature.

\section{Case report}

A 90-year-old Chinese male patient presented a 1-week history of right neck pain and enlargement of the cervical lymph nodes at the Third Affiliated Hospital of Guangzhou Medical University (Guangzhou, China). Physical examination revealed that his general condition was stable with a normal body temperature and typical laboratory results, including blood count, and differential, renal and liver functions. Numerous enlarged right cervical lymph nodes measuring $1.0-4.0 \mathrm{~cm}$ in diameter were detected via palpation of the superficial lymph nodes. A computed tomography scan of the chest and abdomen detected several enlarged lymph nodes, which were located in the mediastinum and right supraclavicular fossa. The patient was considered to have stage III non-Hodgkin's lymphoma and one of the right cervical lymph nodes was surgically removed for histopathological examination. The enlarged lymph node was grey-reddish in color and well-circumscribed to the surrounding tissues. Written informed consent was obtained from the patient prior to the publication of this case report and the accompanying images.

Surgically removed tissue specimens were formalin fixed and paraffin embedded for subsequent analyses. Hematoxylin 

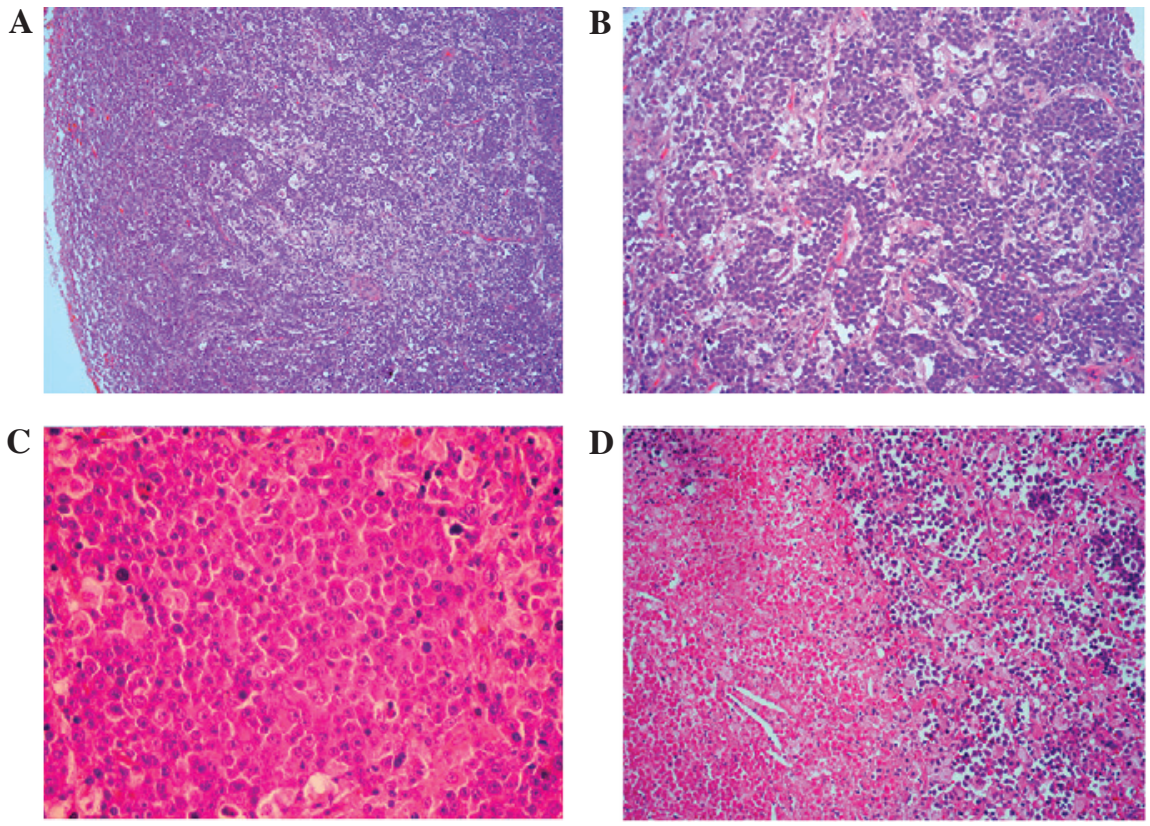

Figure 1. Morphological features of the right cervical lymph node. (A) Diffuse dense lymphoid infiltrate was observed in the cervical mass and the normal architecture of the lymph node was completely destroyed (magnification, $\mathrm{x} 40$ ). (B) There was a diffuse infiltration of large tumor cells with a sinusoidal growth pattern in the cervical lymph node (magnification, x200). (C) Various tumor cells exhibited a large immunoblast-like appearance with round pale nuclei containing large central nucleoli and an abundant cytoplasm, whereas other tumor cells exhibited plasmablastic differentiation with round eccentric nuclei (magnification, 200x). (D) Focal necrosis could be observed in the tumor (magnification, x400). The cervical lymph node was stained with hematoxylin and eosin prior to visualization.

and eosin-stained sections were prepared according to the standard methods. For cytogenetic analysis, DNA was extracted from the paraffinized tissue samples using a DNeasy Blood \& Tissue Kit (Qiagen, Inc., Valencia, CA, USA). T-cell receptor and immunoglobulin gene rearrangement studies were performed. Two sets of primers (tube A, 145-255 bp; tube B, 80-220 bp) were used to amplify the rearranged T-cell receptor- $\gamma$ gene. A T-cell lymphoma case with a known monoclonal rearrangement was used as the positive control, a non-lymphoid and hematopoietic tumor was used as the negative control, and a reaction without template DNA was simultaneously run as the blank control. $\beta$-actin was amplified as an internal control. FRIII-J segmentation were conducted for IgH gene rearrangements. RAJI cells (Sigma-Aldrich, St. Louis, MO, USA) were used as positive controls and a previous negative sample was used as a negative control.

Histologically, the removed specimen resembled a cervical lymph node with effacement of the normal architecture (Fig. 1A), and a diffuse infiltration of large tumor cells with a sinusoidal growth pattern (Fig. 1B). The tumor cells were large with round nuclei, dispersed chromatin, a prominent nucleolus and a moderate eosinophilic or amphophilic cytoplasm. In addition, some of the large tumor cells were immunoblastic-like in appearance, with a single central nucleolus and an abundant cytoplasm, whereas others had a plasmablastic-like appearance, with eccentrically located nuclei. Furthermore, atypical multinucleated neoplastic giant cells, and focal necrotic areas, were observed in the tissue (Fig. 1C and D). Immunohistochemical analyses demonstrated that the large tumor cells were strongly positive for ALK in a granular cytoplasmic distribution, which has previously been described in cases of clathrin/ALK-fusion (Fig. 2A). In addition, the tumor cells demonstrated high levels of expression of CD45, CD138, epithelial membrane antigen (EMA), MUM-1 and lambda (Fig. 2B-E). Conversely, the tumor cells were negative for the expression of the B-cell markers CD20 (Fig. 2F) and CD79a. Furthermore, assays for CD2, CD3, CD7, CD8, CD30, CD38, CD43, CD56, CD68, kappa, granzyme B, terminal deoxynucleotidyl transferase, Pan-cytokeratin, S-100, HMB-45 and Melan-A were all negative. The immunohistochemical analyses were performed using a Dako Autostainer with Envision (+) Detection Kit (Dako, Glostrup, Denmark). Polymerase chain reaction assays were unable to detect clonal rearrangements in the $\mathrm{T}$ cell receptor- $\gamma$ and immunoglobulin heavy chain genes $(\mathrm{IgH})$ in the tumor lesion. Furthermore, in situ hybridization was unable to detect an EBV infection in the lesion. For detection of EBV infection in the tissues, in situ hybridization for EBV-encoded RNAs (EBERs) was performed on biopsy samples using an EBER detection kit purchased from Dako, according to the manufacturer's instructions.

On the basis of the present histopathological and molecular results, the patient was diagnosed with ALK-positive LBCL with plasmablastic differentiation, according to the WHO classification criteria (1). Subsequently, the patient received treatment with the following 21-day chemotherapy regimen: Cyclophosphamide, $750 \mathrm{mg} / \mathrm{m}^{2} \mathrm{~d} 1$; doxorubicin, $500 \mathrm{mg} / \mathrm{m}^{2}$ $\mathrm{d} 1$; vincristine, $1.4 \mathrm{mg} / \mathrm{m}^{2} \mathrm{~d} 1$; prednisone, $60 \mathrm{mg} / \mathrm{m}^{2} \mathrm{~d} 1-\mathrm{d} 5$; and etoposide, $100 \mathrm{mg} /$ day d3-d5. However, clinical deterioration was associated with rapid enlargement of the retroperitoneal lymph node (diameter, $10 \mathrm{~cm}$ ). During the initial cycle of chemotherapy, the patient developed pleural effusions with respiratory distress. A bone marrow examination was performed following the initial cycle of chemotherapy and no abnormality was detected. However, the clinical status of the 

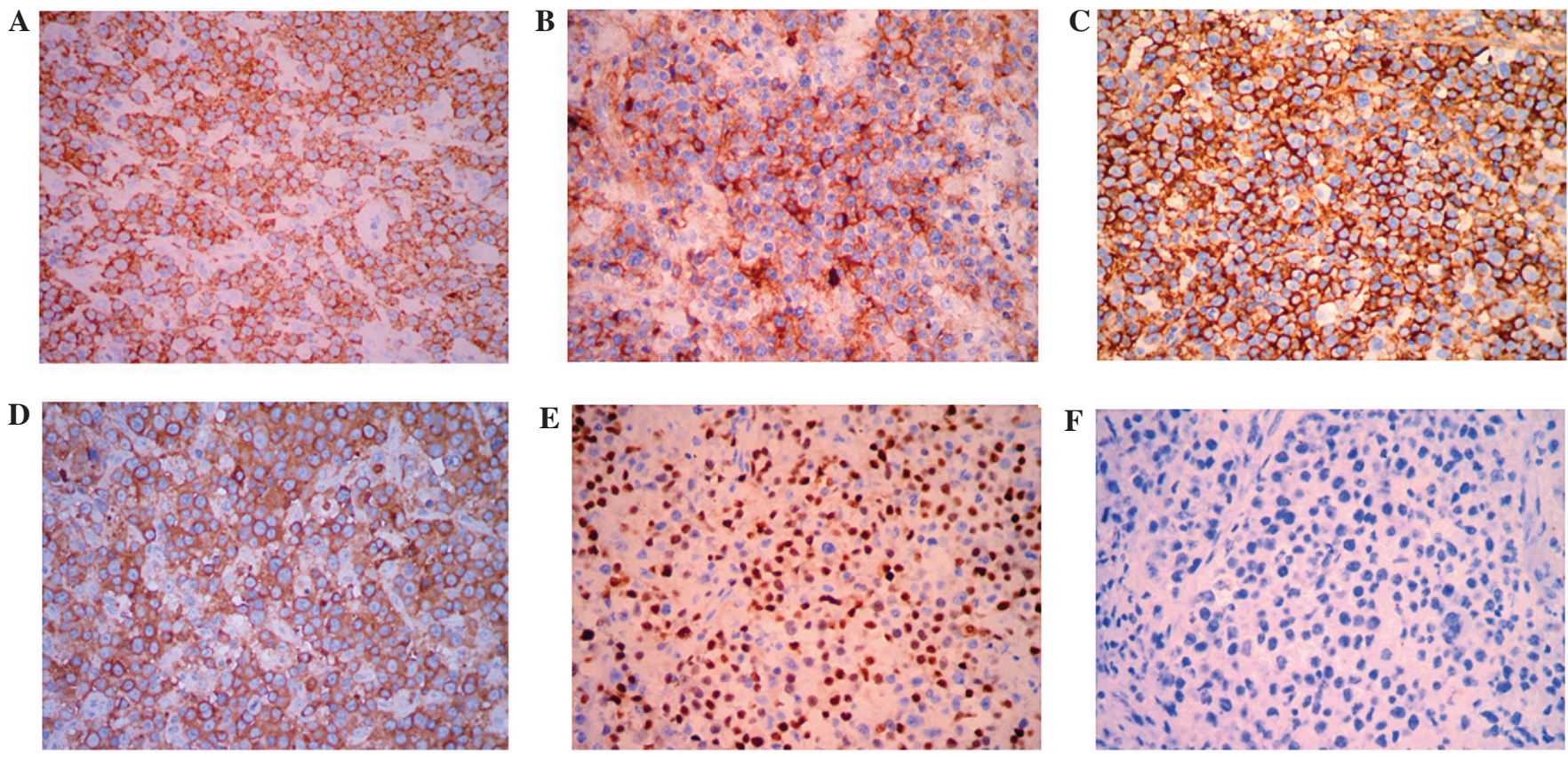

Figure 2. Immunohistochemical analysis of the tumor sample. The tumor cells tested positive for (A) cytoplasmic granular anaplastic lymphoma kinase and the expression of the (B) epithelial membrane antigen, (C) CD138, (D) lambda and (E) multiple myeloma oncogene-1, whereas they tested negative for (F) CD20 expression. The tumor cells were stained with hematoxylin and eosin prior to visualization (magnification, $\mathrm{x} 400$ ).

patient continued to deteriorate rapidly, and he succumbed to the disease within 4 months of initial presentation.

\section{Discussion}

ALK-positive LBCL is a rare subtype of DLBCL, which was recognized by the WHO classification of tumors of hematopoietic and lymphoid tissue in 2001 (4). The diagnosis of ALK-LBCL is typically based on the detection of aberrant ALK protein expression levels in the tumor, which may also exhibit an immunoblastic or plasmablastic morphology, and a lack of CD20, CD79a and PAX5 expression. Since its initial recognition, 50 well-characterized cases of ALK-positive LBCL have been reported in the literature, including a total of twelve pediatric cases (5,6-10). In adult patients, the majority of ALK-positive LBCL tumors occur in males (male/female ratio, 4.3:1), whereas it affects the genders equally in pediatric patients (male/female ratio, 1.4:1) (8). In addition, no significant difference between the mortality rate of male and female patients with ALK-LBCL has been reported (9). Plasmacytic differentiation markers, including CD38, CD138 and VS38c, have been detected in all previously reported cases of ALK-LBCL, and EMA expression has been detected in the majority of the cases. The patient in the present case tested negative for clonal rearrangement in the $\operatorname{IgH}$ gene, and this is consistent with the finding that some B-cell lymphomas do not demonstrate clonal IgH amplification, due to of a lack of consensus target sequences or target site alterations as a result of somatic hypermutation (11). This result suggests that cytogenetic analysis of immunoglobulin genes may not be reliable in the diagnosis of ALK-positive LBCL. Furthermore, consistent with previous studies (1), EBV infection was absent in the present case.

The key histological differential diagnoses include ALK-positive anaplastic large cell lymphoma (ALCL), anaplastic variants of DLBCL, plasmablastic lymphoma, and primary effusion lymphoma, all of which exhibit an ALK-positive immunophenotypic profile or plasmacytic differentiation with CD20-negativity, which may confound the diagnosis of ALK-positive DLBCL. ALK-positive ALCL is typically associated with large tumor cells that exhibit an abundant cytoplasm and pleomorphic nuclei, and elevated expression levels of CD30 on the cell membrane and in the Golgi region (11). Conversely, CD30 expression is typically negative in ALK-positive LBCL, although focal and weak staining has been reported in a few cases of ALK-positive LBCL (11). The expression of ALK and other plasmacytic markers, including CD138, VS38c and MUM-1, and the lack of viral infection, including infections caused by the human immunodeficiency virus, EBV, and human herpesvirus 8, should aid in the diagnosis of ALK-positive LBCL.

ALK-positive LBCL is generally considered a distinct disease entity with an aggressive progression that is associated with a poor prognosis; the overall median survival of patients with advanced stage III/IV ALK-positive LBCL was 11 months in a previous study (9). However, previous studies have reported a prolonged survival rate ( $>10$ years) for patients with advanced stage ALK-positive LBCL $(5,7)$. Of the 50 cases reported in the literature, the clinical stage at disease presentation was the factor most significantly associated with the survival rate of patients with ALK-positive LBCL. Notably, there was no significant difference between the survival rates of pediatric and adult patients, despite reports of more intensive therapies being used for pediatric cases (9). To date, no clinical trials investigating an optimal treatment regimen for ALK-positive LBCL have been reported. As the ALK-positive LBCL tumor cells are typically negative for the CD20 antigen, this tumor is thus insensitive to treatment with rituximab. A previous case reported an example where ALK-positive LBCL was 
treated with rituximab therapy; however, the patient involved succumbed to the disease within 6 months of diagnosis (9). In pediatric patients with ALK-positive LBCL tumors, the majority of regimens used, including the lymphoma malignant B-89, pediatric oncology group 8719, and CHOP protocols, are highly intensive, and only four patients $(33.3 \%, 4 / 12)$ succumbed to relapse or progressive disease at the time of the reports $(3,6,7)$. In the present case, an elderly male patient was diagnosed with stage III ALK-positive LBCL and exhibited rapid clinical decline despite having been treated with the E-CHOP regimen of chemotherapy. The patient eventually succumbed to multiple system organ failure within 4 months of the initial diagnosis, possibly due to chemotherapy intolerance.

In conclusion, we present a rare case of ALK-positive LBCL occurring in an elderly male patient, with rapid tumor progression. To the best of our knowledge, this case is the eldest patient with ALK-positive LBCL to be described in the literature. Previous studies have suggested that some patients with advanced stage ALK-positive LBCL may have prolonged survival rates; however, it is typically associated with high aggressiveness and a poor prognosis, particularly in elderly patients. Further research is required in order to develop novel therapeutic strategies for treating and improving the prognosis of patients with ALK-positive LBCL.

\section{References}

1. Swerdlow SH, Campo E and Harris NL (eds): Chapter 6. In: WHO classification of Tumours of Haematopoietic and Lymphoid Tissues. 4th edition. IARC Press, Lyon, France, pp254-255, 2008.
2. Gesk S, Gascoyne RD, Schnitzer B, Bakshi N, Janssen D, Klapper W, Martín-Subero JI, Parwaresch R and Siebert R: ALK-positive diffuse large B-cell lymphoma with ALK-Clathrin fusion belongs to the spectrum of pediatric lymphomas. Leukemia 19: 1839-1840, 2005.

3. Yin WH, Guo N, Tian XY, Li Y and Li Z: Pediatric anaplastic lymphoma kinase-positive large B-cell lymphoma: A case report and review of the literature. Pediatr Dev Pathol 15: 318-323, 2012.

4. Jaffe ES, Harris NL and Stein H (eds): Chapter 6. In: World Health Organization classification of tumours: tumours of haematopoietic and lymphoid tissues. 3rd edition. IARC Press, Lyon, France, pp214-215, 2001.

5. Delsol G, Lamant L, Mariamé B, Pulford K, Dastugue N, Brousset P, Rigal-Huguet F, al Saati T, Cerretti DP, Morris SW and Mason DY: A new subtype of large B-cell lymphoma expressing the ALK kinase and lacking the 2; 5 translocation. Blood 89: 1483-1490, 1997.

6. Bubała H, Małdyk J, Włodarska I, Sońta-Jakimczyk D and Szczepański T: ALK-positive diffuse large B-cell lymphoma. Pediatr Blood Cancer 46: 649-653, 2006.

7. Isimbaldi G, Bandiera L, d'Amore ES, Conter V, Milani M, Mussolin L and Rosolen A: ALK-positive plasmablastic B-cell lymphoma with the clathrin-ALK gene rearrangement. Pediatr Blood Cancer 46: 390-391, 2006.

8. Beltran B, Castillo J, Salas R, Quiñones P, Morales D, Hurtado F, Riva L and Winer E: ALK-positive diffuse large B-cell lymphoma: Report of four cases and review of the literature. J Hematol Oncol 2: 11, 2009.

9. Gascoyne RD, Lamant L, Martin-Subero JI, Lestou VS, Harris NL, Müller-Hermelink HK, Seymour JF, Campbell LJ, Horsman DE, Auvigne I, et al: ALK-positive diffuse large B-cell lymphoma is associated with Clathrin-ALK rearrangements: Report of 6 cases. Blood 102: 2568-2573, 2003.

10. Adam P, Katzenberger T, Seeberger H, Gattenlöhner S, Wolf J, Steinlein C, Schmid M, Müller-Hermelink HK and Ott G: A case of a diffuse large B-cell lymphoma of plasmablastic type associated with the $\mathrm{t}(2 ; 5)(\mathrm{p} 23 ; \mathrm{q} 35)$ chromosome translocation. Am J Surg Pathol 27: 1473-1476, 2003.

11. Stachurski D, Miron PM, Al-Homsi S, Hutchinson L, Harris NL, Woda B and Wang SA: Anaplastic lymphoma kinase-positive diffuse large B-cell lymphoma with a complex karyotype and cryptic 3' ALK gene insertion to chromosome 4 q22-24. Hum Pathol 38: 940-945, 2007. 